Insights in enzyme functional dynamics and activity regulation by single molecule studies

Jørgensen, Sune Klamer; Hatzakis, Nikos

Published in:

Biophysical Reviews and Letters

DOI:

10.1142/S1793048013300028

Publication date:

2013

Document version

Publisher's PDF, also known as Version of record

Citation for published version (APA):

Jørgensen, S. K., \& Hatzakis, N. (2013). Insights in enzyme functional dynamics and activity regulation by single molecule studies. Biophysical Reviews and Letters, 8(3\&4), 137-160.

https://doi.org/10.1142/S1793048013300028 


\title{
INSIGHTS IN ENZYME FUNCTIONAL DYNAMICS AND ACTIVITY REGULATION BY SINGLE MOLECULE STUDIES
}

\author{
SUNE K. JØRGENSEN and NIKOS S. HATZAKIS* \\ Bio-Nanotechnology Laboratory, Department of Chemistry \\ Nano-Science Center, Lundbeck Foundation Center \\ Biomembranes in Nanomedicine University of Copenhagen \\ 2100 Copenhagen, Denmark \\ *hatzakis@nano.ku.dk
}

Received 18 July 2013

Revised 15 September 2013

Published 14 January 2014

\begin{abstract}
The advent of advanced single molecule measurements heralded the arrival of a wealth of dynamic information revolutionizing our understanding of protein dynamics and behavior in ways not deducible by conventional bulk assays. They offered the direct observation and quantification of the abundance and life time of multiple states and transient intermediates in the energy landscape that are typically averaged out in non-synchronized ensemble measurements, thus providing unprecedented insights into complex biological processes. Here we survey the current state of the art in single-molecule fluorescence microscopy methodology for studying the mechanism of enzymatic activity and the insights on protein functional dynamics. We will initially discuss the strategies employed to date, their limitations and possible ways to overcome them, and finally how single enzyme kinetics can advance our understanding on mechanisms underlying function and regulation of proteins.
\end{abstract}

Keywords: Single-molecule microscopy; enzyme kinetics; allosteric regulation; enzyme protein immobilization; liposomes; conformational selection; induced fit; nanodiscs; protein folding; FRET.

40 Special Issue Comment: This review focuses on functional dynamics of individtime $\square$ ual enzymes and is related to the review on ion channels by Lu, ${ }^{44}$ the reviews on mathematical treatment of Flomenbom ${ }^{45}$ and Sach et al., ${ }^{46}$ and review on FRET by Ruedas-Rama et al. ${ }^{41}$

\section{Contents}

1 Introduction

2 Strategies for Directly Observing Individual Enzyme Turnover 139

2.1 Changes in fluorescent properties of enzyme cofactors . . . . . . . 139 
2.2 Use of prefluorescent substrate analogues for single turnover detection . . . . . . . . . . . . . . . . . 141

2.3 Zero-mode waveguides for single turnover detection . . . . . . . . . . 144

3 State of the Art Immobilization Method for Reliable Single Molecule Studies

3.1 Protein foot for minimized interactions with surfaces . . . . . . . . . 145

3.2 Anti-Brownian electrokinetic trap (ABEL trap) . . . . . . . . . . . 145

3.3 Nanoreactors encapsulation in virus capsid . . . . . . . . . . . . . 146

3.4 Femtoliter reaction vessels for single enzyme readout . . . . . . . . . 147

3.5 Liposomes as novel platforms for single molecule studies . . . . . . . 147

4 Insight from Single Molecule Studies

4.1 Dynamic and static heterogeneities and enzymatic

functional states . . . . . . . . . . . . . . . . . . . . . 149

4.2 Static heterogeneities . . . . . . . . . . . . . . . . . . 152

4.3 Comprehensive understanding of enzymatic regulation by SM studies . . . . . . . . . . . . . . . . . 152

5 Conclusion and Future Directions

\section{Introduction}

The advent of single molecule (SM) techniques in the dawn of the 21st century heralded the arrival of an unprecedented wealth of dynamic information revolutionizing our understanding of protein dynamics and behavior in ways unattainable by conventional bulk measurements. Combined with advanced computational and spectrometric techniques, SM measurements revealed that proteins are not primarily static within accessible conformations, but instead constantly fluctuate in a wealth of time scales sampling multiple interconverting conformations intuitively assumed to exhibit different activities.

The abundances of protein energetically favored conformations and frequencies of fluctuations between them are defined by the multidimensional protein free energy landscape. ${ }^{1-3}$ Pioneer work from Frauenfelder and colleagues insightfully connected this energy-landscape concept to protein function and characterized the features of the landscape ${ }^{1}$ and their dependence on solvents. ${ }^{4}$ A comprehensive description of protein dynamic behavior and function requires a full elaboration of its energy landscape and how it is remodeled by regulatory interactions. Despite great efforts made in this direction, the path to fully understand protein function remains arduous. Molecular dynamic simulations and free energy calculations have provided valuable knowledge on protein folding landscape, internal dynamics and their potential correlation to the catalysis step but are often limited to short millisecond time scales. ${ }^{5-9} \mathrm{SM}$ techniques have emerged as an indispensable tool in the quest of understanding protein function as they are ideally suited for the direct observation and quantification of the activity, abundance and lifetime of multiple states and transient intermediates in the energy landscape that are typically averaged out in non-synchronized ensemble measurements. 
The very first single enzyme experiments were done in the 60ies, when Rotman used fluorescence microscopy to analyze the kinetics of individual $\beta$-D-galactosidase molecules encapsulated in water micro-droplets dispersed in oil and loaded with a prefluorescent substrate analogue. ${ }^{10}$ One seminal finding of these pioneer measurements was that enzymes subjected to a heat shock would either remain fully active or completely denature. This "digital-like" behavior was in stark contrast to ensemble measurements where thermal deactivation resulted in gradual activity reduction and illustrated the power of analyzing individual enzyme kinetics. However, three decades of further technological development in microscopy were required before imaging techniques were sensitive enough to probe the detailed kinetic properties of individual enzymes.

The two principal approaches for directly observing individual molecules are force and fluorescence measurements and combinations thereof. ${ }^{11}$ Force measurements include atomic force microscopy ${ }^{12-16}$ and optical and magnetic tweezers. ${ }^{17-19}$ Fluorescence includes polarization, lifetime, particle tracking, ${ }^{22-27}$ intensity measurements ${ }^{21,28-33}$ and Fluorescence Resonance Energy Transfer (FRET). ${ }^{20,21}$ FRET studies serves as "spectroscopic ruler" 34 providing information on the distance between donor and acceptor fluorophores placed within a certain proximity. FRET has been extensively used to probe conformational dynamics of multiple proteins ${ }^{34-39}$ and has been extensively reviewed elsewhere ${ }^{34,40}$ and in this Special Issue by Angel Orte. ${ }^{41}$ Patch clamp techniques have been extensively used or studies on single ion channels and are reviewed elsewhere, ${ }^{42,43}$ their combination with fluorescent microscopy is reviewed in this issue by Peter Lu. ${ }^{44}$ The techniques used for single molecule detection have been extensively reviewed elsewhere. ${ }^{21,29,32}$ In this review, we will focus primarily on the use of fluorescence microscopy for studying mechanisms of enzymatic activity and the insights gained on enzyme functional dynamics. We will initially discuss the strategies employed to date, their limitations and possible ways to overcome these, then finally how single enzyme kinetic measurements can advance our understanding of protein regulation.

The mathematical and statistical methodology for extracting mechanistic insights from single molecule traces are extensively described elsewhere in this Special Issue by Flomenbom ${ }^{45}$ and by Sachs. ${ }^{46}$

\section{Strategies for Directly Observing Individual Enzyme Turnover}

\subsection{Changes in fluorescent properties of enzyme cofactors}

The first studies at the fundamental limit of individual catalytic turnovers were done on sol gel immobilized cholesterol oxidase (COx) using single molecule fluorescence microscopy. ${ }^{47} \mathrm{Lu}$ et al. used the intrinsic fluorescence properties of the flavin adenine dinucleotide (FAD), which is part of the $\mathrm{COx}$ active catalytic site and switches between its oxidized (E-FAD, fluorescent) and reduced (E-FADH 2 , nonfluorescent) state (Figs. 1(a) and 1(b)). Parking a laser beam on an individual COx enzyme, they recorded the fluctuation of fluorescence through successive enzyme 
(a)

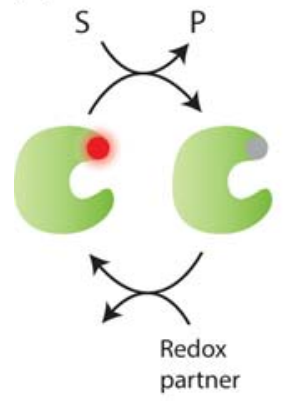

(b)

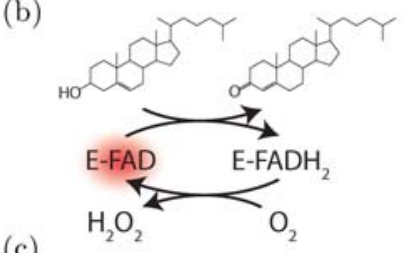

(c)

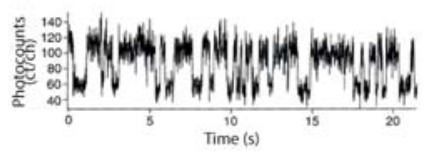

(d)

(e)
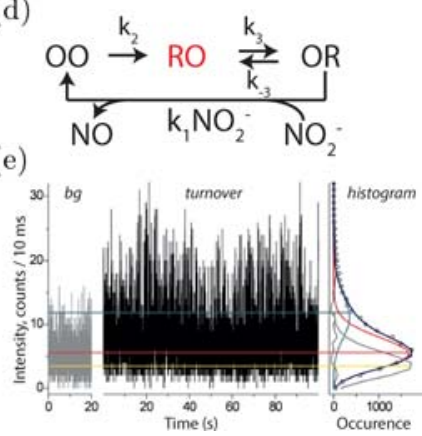

Fig. 1. (a) General description of assays for measuring individual enzyme turnovers exploiting fluorescent property changes of cofactors within the catalytic cycle. (b), (c) Pioneer measurements directly observing stochastic turnover cycles for cholesterol oxidase using FAD cofactor intensity changes. (From Ref. 47. Reprinted with permission from AAAS.) (d), (e) Redox dependent quenching of a chemically attached chromophore allowed direct observation of individual turnovers for nitrite reductase and quantification of the electron transfer rates between the enzyme states.

cycles (Fig. 1(c)). Using the distribution of reaction lifetimes for the high fluorescence state, the authors were able to quantify the enzymatic rate constant and quantify heterogeneities in activity, both in between seemingly identical enzymes of the population (static disorder) ${ }^{48,49}$ and for an individual enzyme over the course of time (dynamic disorder). The time dependent activity fluctuations were proposed to originate from conformational states each of which with a well defined activity. Statistical analysis revealed that the existence of two activity states describes the data adequately. The authors however proposed the possible existence of more than two conformational states or even a broad distribution of conformational states with distinctly different rates for $\mathrm{COx}$.

Variations in the intrinsic fluorescence of flavin cofactors and sol gel immobilization were further exploited in SM functional studies of flavoenzymes. Shi et al. initially identified the existence of distinct static heterogeneities for monomeric dihydroorotate dehydrogenase from Escherichia coli (E. coli), and found them to dominantly depend on the preparation and immobilization method. ${ }^{50}$ The same methodology was later extended to studying the function of homodimeric enzymes of dihydroorotate dehydrogenase ${ }^{51}$ from Lactococcus lactis and hydroxybenzoate hydroxylase ${ }^{52}$ with single turnover resolution. These studies resulted in a detailed kinetic characterization and allowed for the first time the deconvolution of the individual subunit catalytic activity and the cooperativity of dimeric enzymes.

The main limitation of using the properties of redox cofactors to report individual enzyme turnovers is their low quantum yield and photostability that curtails the observation of them to a few seconds. This limits the number of observed turnovers challenging the statistical analysis required to faithfully identify the dynamic nature of individual enzymes. ${ }^{53,54}$ To overcome such limitations Kuznetsova et al. employed the redox state dependent quenching of a biochemically attached photostable chromophore on nitrite reductase. ${ }^{55}$ Nitrite reductase from Aspergillus niger is a 
homotrimer with two copper centers per monomer, each involved in the catalytic reaction. Within each turnover cycle an electron is accepted from a donor molecule by the type 1 copper center, relayed to the type 2 copper center, which uses it to reduce nitrite $\left(\mathrm{NO}_{2}^{-}\right)$to nitric oxide $(\mathrm{NO})$ (Fig. 1(d)). The copper cofactors are not fluorescent but their oxidized state efficiently quenches the site-specific coupled chromophore Atto-655 (Fig. 1(e)). Changes in Atto fluorescence over time are thus directly related to enzymatic turnovers. This approach required site-specific mutation, biochemical labeling, characterization and purification as compared to simply using intrinsic flavin groups, but it also extended the observation times to multiple seconds (Fig. 1(e)), significantly improving statistical analysis. It thus allowed for the, impossible in bulk kinetics, direct quantification of the electron transfer rates between the types 1 and 2 copper centers to be $k_{3}=21 \pm 6 \mathrm{~s}^{-1}$ and $k_{-3}=14 \pm 4 \mathrm{~s}^{-1}$ as well as their heterogeneities in activity. These heterogeneities were postulated to originate from local variations in the coordination spheres of the copper centers.

Chromophore quenching was furthermore used to study the kinetics of dihydropholate reductase (DHFR) and reveal conformational changes in the enzymeligand complex, which were masked in the ensemble stopped-flow studies. ${ }^{56,57}$ To do this the authors coupled the fluorescent dye Alexa-488 at the top of the structural loop of DHFR, which closes after binding of the substrate causing quenching of the fluorescence. The fluorescence changes in Alexa-488 allowed the authors to directly observe substrate binding and conformational changes of the enzyme along the reaction coordinate. Interestingly, even though the enzymatic behavior could be interpreted on the basis of a simple two-state model, the authors anticipated the existence of multiple functional states. Variations in fluorescent properties of chromophores along the catalytic cycle constitute a convenient methodology for SM studies and advances in photostable chromophore synthesis ${ }^{33,58,59}$ could render it applicable to studying a spectrum of oxidoreductases with single turnover resolution. To date, it remains overshadowed by the emergence and use of prefluorescent substrate analogues.

\subsection{Use of prefluorescent substrate analogues for single turnover detection}

The realization of prefluorescent substrate analogues extended the observation time of individual turnover experiments up to two orders of magnitude (several minutes). The underlying principle is simple: the substrate is non-fluorescent and therefore invisible for fluorescent microscopy. Upon enzymatic reaction it is converted to a highly fluorescent product (Fig. 2(a)). Fast product diffusion out of the enzyme active site and the illumination volume allow observation of stochastic individual turnover cycles. Being limited only by background increases and substrate depletion, this method offers unprecedented rich statistical information over extended time scales crucial for deciphering the enzyme function. 


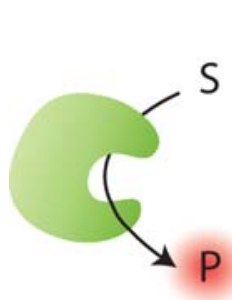

(a)

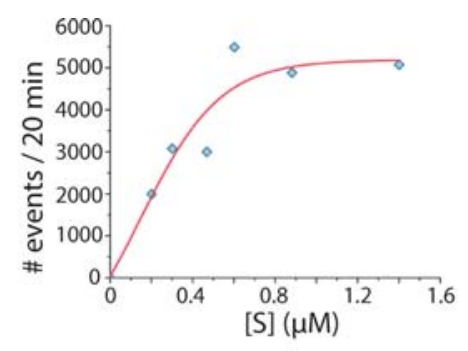

(b)

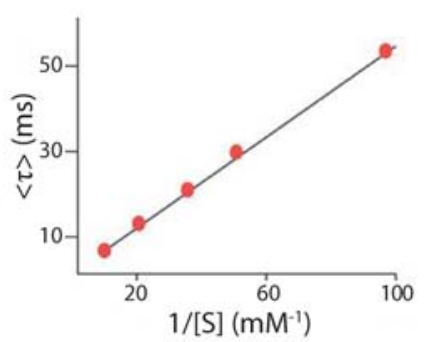

(c)

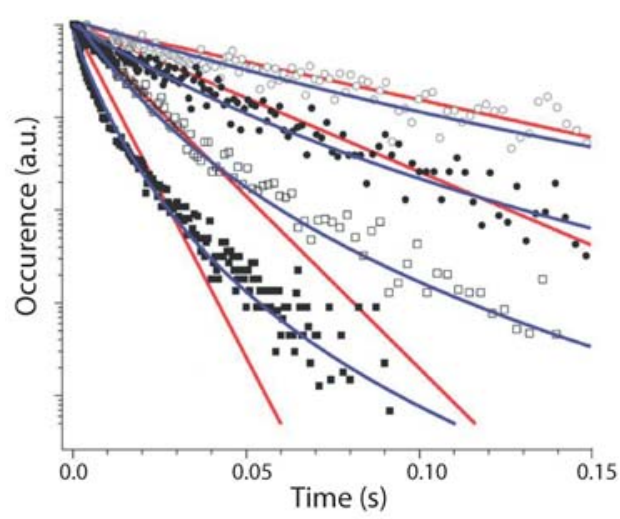

(d)

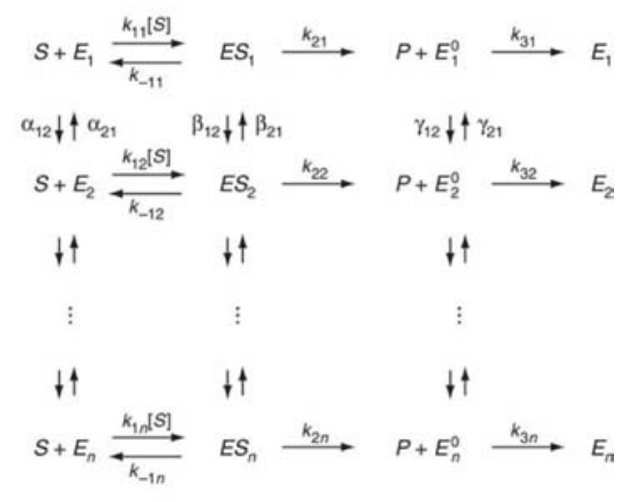

(e)

Fig. 2. (Color online) Prefluorescent substrate analogues for prolonged observation of enzymatic turnovers. (a) The non-fluorescent substrate is enzymatically converted to a fluorescent product, which before diffusing away from the enzyme and detection volume of the microscope allows observation of individual enzymatic turnovers. (b) Measurements on CALB revealed the enzyme to follow the typical substrate dependent saturation behavior as predicted by Michaelis-Menten kinetics. (Reproduced from Ref. 61 with permission, Copyright (c) 2005 WILEYVCH Verlag GmbH \& Co. KGaA, Weinheim.) (c) Single molecule Lineweaver-Burk plot showing $\beta$-galactosidase to follow Michaelis-Menten kinetics. (d) Waiting time distribution of $\beta$-galactosidase enzymes change from monoexponential (red fits) at low substrate concentration to multiexponential (blue fits) at high substrate. (e) Generic multistate model proposed to underlie enzyme function. Enzymes are proposed to sample multiple interconverting conformations with different activities. ((c), (d), (e) reprinted with permission from Macmillan Publishers Ltd: [Nature Chem. Biol.] (Ref. 66), copyright (2006).)

The first experiments with a prefluorescent substrate were performed on horseradish peroxidase (HRP). ${ }^{60}$ The enzyme was immobilized on a glass slide (via biotin/avidin interaction) and the dihydrorhodamine 6G was used as a substrate that upon enzymatic reduction produced the highly fluorescent product rhodamine 6G. Using fluorescence intensity autocorrelation analysis, HRP activity was found to fluctuate over a wide range of time scales. These fluctuations were found to primarily originate from a large distribution of rates for the formation of the enzyme-product complex, rather than the dissociation of product from the enzyme. Though the relatively low signal to noise ratio and limited time measurements complicated the data 
analysis, this study paved the way for later studies using prefluorescent substrate analogues to monitor enzyme function for prolonged periods of time.

Titrating the concentration of prefluorescent analogue, Velonia et al. found an individual lipase B from Candida Antarctica $(C A L B)$ to follow typical substrate dependent saturation behavior which is similar to that observed for enzymes in solution (Fig. 2(b)). ${ }^{61}$ The long time traces furthermore provided a high number of turnovers that was critical for rigorous statistical analysis. The reaction time distribution was accurately fitted with a stretched exponential decay showing the existence of dynamic heterogeneities on the reaction rate. Using a combination of reaction time distribution and autocorrelation analysis the authors concluded the existence of a broad spectrum of conformational states that are coupled and calculated their average life time to be $\sim 35 \mathrm{~ms} .{ }^{61,62}$ They thus proposed a fluctuating enzyme model which involves a spectrum of enzymatic conformations that interconvert on the time scale of the catalytic activity. ${ }^{61,62}$ Using prefluorescent substrate analogues for more lipases showed time dependent activity fluctuations to be a generic lipase phenotype. ${ }^{28,63,64}$

Prolonged observation of individual turnovers for $\alpha$-chymotrypsin allowed the direct observation of the stepwise deactivation of the enzyme. ${ }^{65} \alpha$-chymotrypsin was directly observed to oscillate between active and inactive states with lifetimes of hundreds of seconds and furthermore to switch back to the active state in a lower activity state. The authors proposed that while residing on the inactive state the enzyme is susceptible to important structural changes that upon switching back to its native state allows it to sample a new functional state.

The studies of English et al. established that the Michaelis-Menten equation, the cornerstone of modern enzymology, still holds for single molecules, independent of the number of functional states the enzyme samples. ${ }^{31,66}$ They studied with single turnover resolution the activity of $\beta$-galactosidase and used the waiting times between individual turnovers to plot the Lineweaver-Burk plot and found the anticipated linear dependence (Fig. 2(c)). Importantly, they found the waiting time distribution for low substrate concentrations to decay monoexponentially. At increased substrate concentrations the authors used a multiexponential to fit the data (Fig. 2(d)). At low concentrations, enzyme substrate binding is the ratelimiting step, which yields a pseudo-first order rate constant and therefore produces a monoexponential waiting time distribution. As the substrate concentration is increased, the rate-limiting step is no longer the enzyme substrate binding but rather the conversion of substrate to product. The non-monoexponential decay of the waiting time distribution therefore shows the existence of more than one functional state with lifetimes similar to or slower than the turnover rate. The data were therefore interpreted as originating from interconversion of the enzyme to a spectrum of conformations each of which had different activity (Fig. 2(e)). This is not surprising for $\beta$-galactosidase, which is composed of four monomers that could catalyze independently of each other, however it would be very interesting to 
develop a method to deconvolute and analyze the number of functional states for each monomer.

In general single molecule studies propose the conformational fluctuations of enzymes to lead to multiple Michaelis Menten ES and Product EP complexes and their related transition states. ${ }^{66,67}$ However the number of states, their activity and interconversion rates are challenging to be accurately determined. In fact in multiple cases the same experimental data are interpreted using contrastingly different models (see also Sec. 4.2). Advances in data treating techniques, ${ }^{62,68,69}$ some of which are presented in this Special Issue, provide means to unambiguously convert experimental data to a unique mechanism that can generate them.

The advance in our understanding of enzyme behavior by employing prefluorescent substrate analogues has been staggering and has opened up new fields of biological inquiry. The main remaining bottleneck is the limited number of available substrates and the presence of two cleavable groups in some of them, which requires careful consideration in the data evaluation and treatment. The future of these studies will thus depend on the development of novel prefluorescent substrate analogues for multiple biocatalysts.

\subsection{Zero-mode waveguides for single turnover detection}

The realization of zero-mode waveguides (ZMWs) to confine individual biomolecules and interrogate their function opened up new windows to connect optical analysis with biomolecular systems. ${ }^{70}$ Light intensity away from a zero-mode waveguide illuminated by a laser beam from below decays exponentially. The observation volume is thus reduced to the zeptoliter $\left(10^{-21} \mathrm{~L}\right)$ range allowing measurements at micromolar chromophore concentration (ligand or substrate), which is impossible for current conventional fluorescent microscopy setups. Concomitantly ZMWs offer massive parallel single molecule readout at high temporal resolution $(\sim \mathrm{ms})$. This methodology allowed the interrogation of the catalytic behavior of DNA polymerase with single turnover resolution ${ }^{71}$ and also the real time DNA sequencing from single polymerase molecules. ${ }^{72}$ Using four distinguishable fluorescently labeled deoxyribonucleoside triphosphates, DNA polymerases could be continuously observed performing uninterrupted template-directed synthesis over thousands of bases. The high quality data of the method allowed the direct observation of distinct polymerization states and pause sites that corresponded to the secondary DNA structure.

ZMWs are ideal for studying a plethora of dynamic systems ranging from enzyme function, ${ }^{71^{-73}}$ membrane properties ${ }^{74}$ and biological processes. ${ }^{75}$ Their main challenge limiting their wider application is the difficult micro fabrication techniques required to make arrays of nanowells. Undoubtedly, this method has a broad application especially to the study of translation and may allow the observation of translational events involved in the regulation of protein synthesis, such as frame shifting. 


\section{State of the Art Immobilization Method for Reliable Single Molecule Studies}

The Achilles' heel of the majority of single enzyme measurements is the requirement for the direct immobilization of the individual macromolecules in the microscope detection volume. Indeed, several immobilization methods has been employed including non specific adsorption to glass slides, ${ }^{61}$ site specific single-point attachment on glass, ${ }^{55}$ use of porous agarose type gels, ${ }^{50-52,65,66}$ biotin mediated coupling ${ }^{56,57,60}$ and coupling to polystyrene beads. ${ }^{66}$ Though the precise effect of immobilization method and interaction with microenvironment on protein function is scarcely studied, multiple cases over the last year revealed deleterious interactions that might severely impair protein function. The position and length of the tether used to immobilize proteins and nucleic acids can severely impair both their folding times and folding mechanism. ${ }^{76,77}$ Similarly, the functional properties of the membrane bound dihydroorotate dehydrogenase from $E$. coli were found to significantly depend on the presence of detergents ${ }^{50}$ in the sol gel. The dynamics of the photosynthetic antenna protein, allophycocyanin were found to be different in solution ${ }^{78}$ than when immobilized in agarose or to glass. ${ }^{79}$ Lastly, the large static disorder of CALB was attributed to the non specific interactions to the glass surface. ${ }^{61}$ Therefore, over the last years the community is striving to develop methodologies to spatially confine the enzymes in non-intrusive biologically relevant environments. ${ }^{28,29,80,81}$ Here we will briefly review some of these methods.

\subsection{Protein foot for minimized interactions with surfaces}

To avoid non specific adsorption of enzymes to the glass surface and minimize possible artifacts arising from labeling the enzyme, Hatzakis et al. developed an approach to site-specifically immobilize enzymes on surfaces using a "protein foot". 63 Using "click chemistry" 82-85 the lipase from Thermomyces lanuginosa (TLL) was site specifically coupled to bovine serum albumin (BSA). The high affinity of BSA to hydrophobic surfaces allowed the TLL-BSA heterodimer to orient itself with the BSA toward the hydrophobic glass leaving the lipase free in solution. This elegant approach may require laborious chemical synthesis of the linkers for the click chemistry but offered biocompatibility and high reproducibility of the measured kinetic rates along with low static heterogeneities. The authors found the activity of the BSA-TLL heterodimer in solution to be twice as high as the monomeric TLL, indicating that the hydrophobic BSA upregulates the lipase activity by stabilizing the open lid state and redistributing the conformational equilibrium towards the open and active state.

\subsection{Anti-Brownian electrokinetic trap (ABEL trap)}

To avoid any immobilization of enzymes for single molecule studies, the group of Moerner developed the anti-Brownian electrokinetic trap (ABEL trap). ${ }^{86}$ This 
methodology enables extended investigation of solution phase biomolecules through real-time electrokinetic feedback. The idea of the trap is rather simple: The trap monitors the Brownian motion of a nanoparticle by using fluorescence microscopy and then applies a feedback voltage to a microfluidic cell so that the resulting electrokinetic forces produce a drift that exactly cancels the Brownian motion. ${ }^{78}$ This methodology allowed the action mechanism of nitrite reductase to be deciphered ${ }^{78}$ and revealed pathways of interstate dynamics and light-induced conformational changes in the allophycocyanin antenna protein. ${ }^{87}$ Importantly, using the ABEL trap Bockenhauer et al. directly observed for the first time the inherent dynamics of individual $\beta 2$-Adrenergic $\mathrm{G}$ protein coupled receptors. ${ }^{88}$ Their measurements revealed that the receptor samples a diversity of conformational states interconverting in the time scale of milliseconds, providing new insights in the GPCR field. These measurements however were performed in non-native conditions, using detergent to solubilize the transmembrane receptor.

ABEL trap is a promising method for trapping individual biomolecules but several requirements constrain the choice of system to study ${ }^{81}$ : buffers with low conductivity optimized for each system, careful passivation of the surface to minimize non specific binding, and a bright photostable chromophore for accurate feedback and even then most biomolecules remain trapped only for a few seconds. ${ }^{78,81,87}$

\subsection{Nanoreactors encapsulation in virus capsid}

Inspired by the compartmentalization employed by nature where enzymes are often confined in ultra small cellular compartments, the group of Nolte utilized the interior of a virus capsid to encapsulate individual HRP enzymes. ${ }^{90}$ To do this, they used the cowpea chloritic mottle virus (CCMV) that after removal of its RNA provides an empty capsid with an outer diameter of $28 \mathrm{~nm}$ and inner diameter of $18 \mathrm{~nm}$. Using the reversible pH-dependent assembly/disassembly of the CCMV capsid and an appropriately low concentration of HRP they obtained virus capsids containing individual HRP enzymes. The virus capsids were non specifically absorbed onto a glass surface, where the capsid protected the enzyme from directly interacting with the glass surface. Using confocal fluorescent microscopy and a prefluorescent substrate analogue they could observe the activity of individual HRP molecules. Fluorescent autocorrelation analysis showed 3 orders of magnitude slower diffusion of product for the CCMV encapsulated enzyme as compared the diffusion in solution ( $\tau=19.5 \mathrm{~ms}$ versus $\tau=0.04 \mathrm{~ms}$ respectively). The authors took great care to overcome the slow diffusion of product away from the detection volume, which limits the single turnover readout, by increasing the $\mathrm{pH}$ of the solution enlarging the pores of the virus, or by attaching HRP non specifically on the outside of the virus. In doing so they increased 3 fold the diffusion coefficient of the product. The same team, in an attempt to increase the encapsulation efficiency and confine multiple enzymes per capsid, engineered lipases to form coiled coil complexes with the virus capsid protein dimer, which were subsequently assembled to form the virus capsid. ${ }^{91}$ 


\subsection{Femtoliter reaction vessels for single enzyme readout}

Tan et al. employed nanoscopic vials to form independent reactors of femtoliter volumes to confine lactate dehydrogenase and monitor the activity in a parallelized manner ( 100 enzymes simultaneously). ${ }^{92}$ The group of Walt greatly optimized and exploited the use of femtoliter-sized reaction vessel arrays ${ }^{93-96}$ to study enzymatic function. They used soft lithography to prepare wells from polydimethylsiloxane to confine the HRP or the tetrameric $\beta$-galactosidase enzymes and their prefluorescent substrate analogues. ${ }^{93,96,97}$ Using a glass fiber bundle and a custom-made epifluorescence microscope they recorded the activity of hundreds of individual enzymes simultaneously. Their experiments with the $\beta$-galactosidase inhibitor revealed that the tetrametric enzyme exists predominantly in two states, free or completely occupied with four D-galactal inhibitor molecules. They proposed that inhibitor binding to an enzyme induces conformational changes that result in a different enzyme activity each time the inhibitor dissociates. ${ }^{96}$ The slow temporal resolution of a few to tens of seconds prevented observations of time-dependent activity fluctuations, occurring in the millisecond time scale, and thus these measurements provided the average turnover activity over the integration time for these enzymes. Interrogating simultaneously hundreds of individual $\beta$-galactosidase enzymes allowed the first high statistic quantification of static heterogeneities in their activity. ${ }^{93}$ The wide rate distribution of the individual $\beta$-galactosidase molecules was found to originate from variability in the enzyme product-forming rate constant, $k_{c a t}$, rather than the Michaelis-Menten constant, $K_{m}$. The static heterogeneities were attributed to distinct long lived activities of the enzyme, however transitions between such long lived states were not directly observed.

The use of femtoliter reaction vessels is not a panacea for the challenges of singlemolecule assays. Several factors constrain the wide applicability of the assay: The low temporal resolution used ( 2 to $15 \mathrm{~s}$ ) restricts observation of most conformational and functional protein transitions, the observation time is often limited to a few minutes due to substrate depletion, and it requires state of the art lithography surface patterning and imaging techniques. However, despite these technical difficulties, the ability to perform highly parallel measurements of many individual enzymes at the same time renders the approach very powerful, and allows for acquiring novel information about enzymatic behavior.

\subsection{Liposomes as novel platforms for single molecule studies}

Liposomes are ideal systems to spatially confine biomolecular elements in a biocompatible environment and study their behavior. ${ }^{98,99}$ Consisting of phospholipids, the natural constituent of cell membranes, they may be considered as "native-like" membrane systems. Surface tethering of a vesicle allows the spatial localization of few encapsulated macromolecules while keeping them under physiological conditions, far from interactions with a solid substrate that may impair stability and function. Liposomes ${ }^{98}$ may offer (a) the exterior part of the bilayer to act as a 


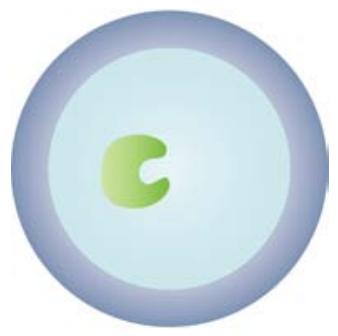

(a)

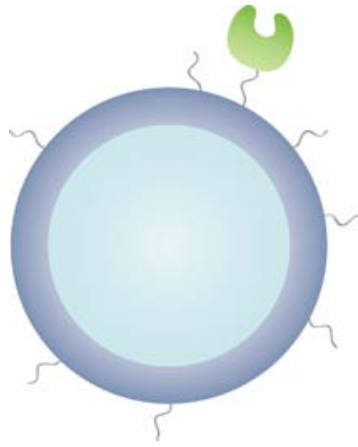

(b)

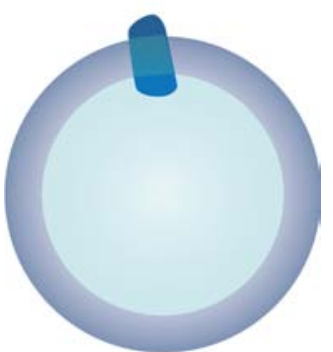

(c)

Fig. 3. Liposomes as versatile biocompatible 3D scaffolds can spatially confine biomolecular entities for single molecule functional and structural studies. Liposomes allow (a) encapsulation, (b) anchorage via flexible linkers of water-soluble proteins, or (c) reconstitution of transmembrane proteins. Development of high-density microarrays of biomimetic scaffolds such as liposomes or nanodiscs for single molecule studies could be a novel way to implement screening of biochemical properties, molecular function, or protein-effector interaction.

biocompatible scaffold to anchor biomolecular elements and study the parameters regulating their spatial localization and function, ${ }^{100-102}$ (b) their membrane to reconstitute membrane proteins and study their function and dynamics ${ }^{89,103,104}$ or (c) their interior to encapsulate biomolecular elements acting as sub-attoliter nanocontainers ${ }^{110^{-112}}$ or perform catalytic reaction using them as miniaturized nanoreactors $^{105-109}$ (Figs. 3(a)-3(c)).

The adaptation of liposomes to single-molecule techniques opens room for assays where a specific number of molecules must be confined within a small biocompatible volume. Initial studies showing the diffusion of individual proteins encapsulated inside the liposomes to be similar to that in solution, ${ }^{113}$ and not to be slowed by transient interaction with the lipid wall, paved the way for using liposomes as nanocontainers for single molecule studies. Later studies exploiting liposomes directly observed folding of single proteins and nucleic acids ${ }^{114-116}$ as well as evaluated the protein folding landscape. ${ }^{117}$ Importantly, the use of liposomes revealed that confinement of individual proteins in ultra small volumes increased protein-ligand interactions by two orders of magnitude. ${ }^{118}$ Such an enhancement was anticipated to be reminiscent of a general mechanism used in cells to increase the efficiency of vital biological reactions by "molecular crowding" or "compartmentalization". 119

Liposome encapsulation of single enzymes is widely recognized as an elegant method, however it comes with its own caveat; the lipid bilayer acting as a barrier limiting substrate/ligand access to the encapsulating enzyme. To overcome this limitation the community has employed smart methodologies such as the use of pore forming toxins, ${ }^{118}$ nested systems of lipid vesicles, part of which release their content during a thermotropic phase transition, ${ }^{120,121}$ or even the use of polymersomes 
permeable to substrate molecules. ${ }^{122}$ Recently, Christensen et al. created highdensity surface-based arrays of small unilamellar liposomes loaded with enzymes and promoted their controlled fusion and content mixing with a second population of diffusing liposomes loaded with enzyme substrate. ${ }^{105}$ Implementation of such ultra-small-volume platforms for single molecule studies could provide novel ways to obtain massive parallel screening of biochemical properties with single molecule resolution.

We recently exploited the use of surface-tethered liposome arrays as a 3D biocompatible scaffold to anchor enzymes via flexible linkers and interrogate their function with single turnover resolution ${ }^{64}$ (Fig. 3(b)). This methodology allows the enzyme to freely sample the solution and its substrate while minimizing non specific interactions with hard matter that may alter its function. We used lipases the workhorse enzymes catalyzing the enantioselective hydrolysis of esters in solution ${ }^{123-126}$ and widely employed for multiple biotechnological applications. ${ }^{124,127}$ Enzymatic activity was measured by confocal fluorescent microscopy using the prefluorescent substrate analogue carboxy fluorescein diacetate, CFDA, which upon hydrolysis provide the highly fluorescent product carboxyfluorescein. The biocompatibility of the approach permitted reproducible measurements of single enzyme kinetics and thorough investigation of parameters underlying its regulation, which will be further discussed in Sec. 4.3.

The versatility and convenient reconditioning of the composition, dimension and properties of individual liposomes would enable their use for multiple applications in single molecule enzymology. We anticipate that high-density surface-based arrays of small unilamellar lipid vesicles ${ }^{100,105,128-130}$ may be employed to spatially confine practically any biocatalyst and investigate protein-ligand or protein-membrane interactions as well as the behavior of regulated or not enzymes with single molecule resolution (Fig. 3). Importantly, liposomes would constitute an elegant approach to reconstitute and investigate the single molecule behavior of multiple transmembrane proteins and enzymes ${ }^{89,104}$ (Fig. 3(c)) in environments resembling "nativelike" conditions, thus overcoming limitations arising from the current methodology based on detergent solubilization. ${ }^{36,37,88}$

\section{Insight from Single Molecule Studies}

\subsection{Dynamic and static heterogeneities and enzymatic functional states}

The combined readouts of advanced molecular dynamic simulations, discussed in this Special Issue by Sachs, ${ }^{46}$ NMR and single molecule FRET experiments provided an incredible wealth of dynamic information of protein dynamics and behavior that would not be deducible from the static crystal structures. These studies revealed that proteins are not static but constantly fluctuating in a wealth of time scales sampling a wide distribution of interconverting conformations and furthermore introduced the notion of conformational energy landscapes of proteins. ${ }^{1-3,131-135}$ The 
first single molecule activity studies of single enzymes kinetics on the other hand unveiled time dependent activity fluctuations occurring in the same time scale as conformational motions. ${ }^{47}$ Naturally, these activity fluctuations were interpreted as originating from protein conformational fluctuations. ${ }^{1,2,31,67,135,136}$ Indeed, even though the first single turnover observations of glucose oxidase and dihydropholate reductase were sufficiently interpreted by simple models of two functional states, ${ }^{47,56,57,137}$ the authors postulated the existence of more complex behavior and the existence of multiple states. ${ }^{56,57}$ It was thus proposed that enzymes sample a wide distribution of interconverting conformations along the conformational landscape each of which had different activity. ${ }^{28-30,138,139}$

It is becoming increasingly apparent however that simpler models with a discrete number of activity states may equally well describe the experimental observations for most of the enzymes. ${ }^{64,137,140-143}$ Terentyeva et al. explored the inherent limitations and artifactual conclusions that may arise from treating trajectories of individual enzymatic turnovers. To do this they performed a systematic comparison of the commonly used binning and thresholding method with change point analysis using both simulated time traces of varying intensity or signal to noise ratios and published data on $\alpha$-chymotrypsin. Their results for $\alpha$-chymotrypsin revealed that the concave shape of the waiting time distribution, often fitted with a stretched exponential and used as the hallmark of a distribution of functional states, may possibly originate from artifactual analysis procedure. ${ }^{142,143} \alpha$-chymotrypsin was found not to sample multiple functional states, but rather to have a single activity over time. They furthermore highlighted the difficulties to accurately determine the number of exponentials underlying the waiting time histograms, if the number of exponentials needed for the fit is more than three.

Correctly identifying the mechanism of enzymes from noisy binary time series is an omnipotent limitation of single molecule data. To overcome these limitation Flomenbom et al. developed a pioneer method to filter noise using numerical algorithm with various special statistical treatments that is based on a general likelihood function, ${ }^{68,69}$ also reviewed in this issue. In addition, using canonical forms of reduced dimensions ${ }^{69,144}$ allowed deducing the underlying multisubstate on-off kinetic scheme from the statistical properties of a binary trajectory with significantly improved accuracy than other methodologies.

We recently found multiple enzymes to sample two functional states rather than a distribution of them (Fig. 4). We acquired data for two lipase variants, ${ }^{64}$ lipase from Thermomyces Lanuginosus (TLL) and Candida Antarctica and obtained published activity traces for $\beta$-galactosidase from $E$. coli $^{66}$ for the nitrite reductase from Alcaligenes faecalis ${ }^{55}$ and for bovine $\alpha$-chymotrypsin, ${ }^{65}$ practically covering the spectrum of enzymes that have had their activity investigated at the single turnover resolution with high statistics. In agreement with earlier studies $\beta$-galactosidase and $\alpha$-chymotrypsin could not be fitted with a two state model. This is expected for $\beta$-galactosidase since it consists of four monomers that could catalyze independently of each other. Importantly, we found the waiting time distribution of 


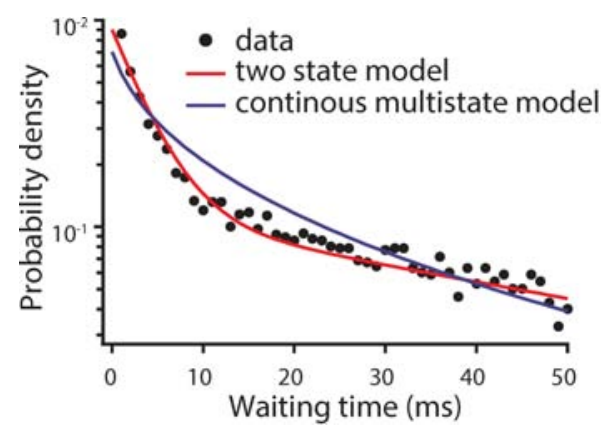

(a)

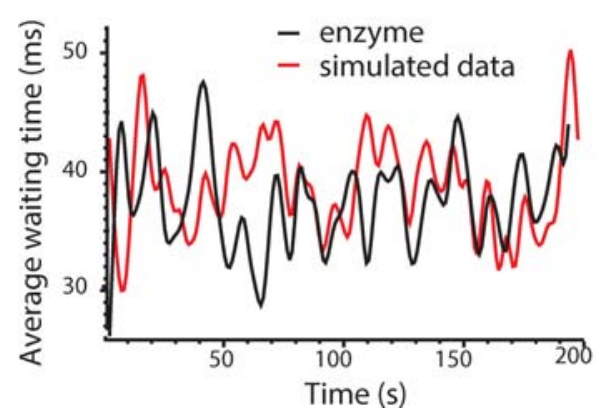

(c)

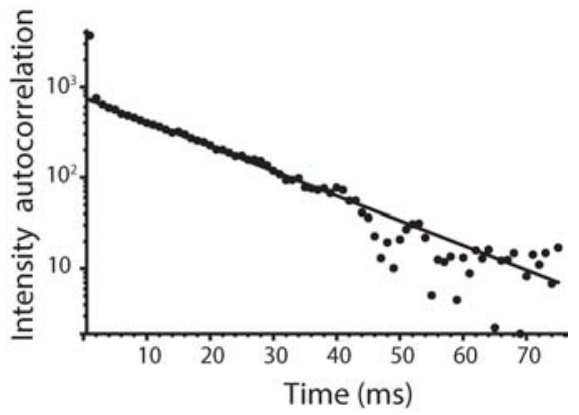

(b)

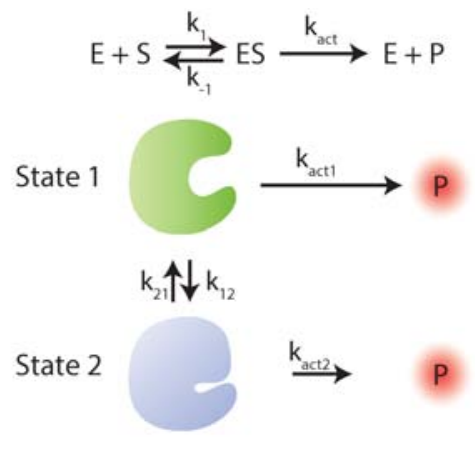

(d)

Fig. 4. A discrete number of functional states rather than a continuous distribution of them accurately describe the behavior of multiple enzymes. (a), (b) Histogram of waiting time distribution and intensity autocorrelation of TLL is more accurately described by two functional states rather than a distribution of them. (c) Activity fluctuations in multiple time scales (millisecond to second), widely interpreted to originate from a wide distribution of functional states is accurately described by the existence of just two functional states. (d) Schematic representation of a general two-state model that accurately describes the catalytic behavior of multiple enzymes. The model is an extension of the Michaelis-Menten equation and contains two interconverting functional states with respective activity rates $k_{\text {act } 1}$ and $k_{\text {sact } 2}$. (Reprinted with permission from Ref. 64 . Copyright (2012) American Chemical Society.)

both lipases and nitrite reductase to be accurately fitted with a double exponential decay and furthermore so, significantly better than the stretched exponential decay previously employed to describe multistate activity models (Fig. 4(a)). Similarly, both the waiting time and intensity autocorrelation showed monoexponential decay consistent with the presence of two functional states (Fig. 4(b)). The intensity autocorrelation being independent of thresholding eliminates artifacts arising from such data treatment ${ }^{142,143}$ and provides a solid validation of the presence of the two functional states. The two functional states were also found to accurately describe TLL's activity fluctuation in multiple time scales (millisecond to second), a phenotype widely interpreted to be the consequence of a wide distribution of activity rates (Fig. 4(c)). ${ }^{28,29,47,61-63,65,66,145}$ The existence of a small number of 
discrete functional states was also directly observed by pioneer measurements on lysozyme, ${ }^{140,146}$ further indicating this to be a generic phenotype underlying the behavior of multiple enzymes (Fig. 4(d)).

\subsection{Static heterogeneities}

Static disorder, the heterogeneous activity between individual enzymes of a population, is an important phenotype underlying protein behavior that was unveiled by SM studies. Multiple theories have been developed to explain its underlying molecular origin, assuming it does not originate from non-native interactions with the assay medium. ${ }^{50,61,76,77,79}$ The two prevailing theories rely either on the existence of chemical heterogeneities or on the existence of long-lived conformational states in the protein energy landscape. The chemical heterogeneities hypothesis was proposed by studies on glucose oxidase, suggesting that static disorder originates from proteolytic damages on the protein ${ }^{47}$ and from studies on alkaline phosphatase postulating that it originates from posttranslational modifications. ${ }^{147}$ The longlived conformational states hypothesis on the other hand was initially proposed from experiments on lactate dehydrogenase. ${ }^{48,92}$ Measurements on $\beta$-galactosidase examined further static disorder and proposed that it practically is a slow dynamic disorder, originating from rare transitions between states with different activity $k_{\text {cat }}$. However, the low frequency of such transitions renders their direct observation challenging or impossible with current state of the art methodologies and to date they have not be recorded.

Single molecule FRET studies on the other hand directly observed individual ribozyme molecules to fold into multiple distinct native states with different catalytic activity. ${ }^{148}$ The authors examined if these states correspond to (a) folded states of ribozyme molecules that have different sequences (errors in synthesis, unintended chemical modifications or degradation) and thus originate from chemical heterogeneities between Ribozymes or (b) multiple distinct folds of the same sequence, and thus originate from multiple energy minima in protein landscape. Their findings show interconversion between these states in ultra slow time scales ( $9 \%$ of molecules in $40 \mathrm{~min}$ observation times) supporting that static heterogeneity at least for Ribozyme originates from slow dynamic disorder. ${ }^{148}$ It would be enticing to directly observe such transitions for enzymes and decipher whether indeed they have multiple local minima corresponding to native, rarely interconverting, functional states and furthermore how they are controlled by regulatory cofactors. Capturing such transitions in proteins would entail methodologies that combine high throughput analysis with millisecond time resolution and long-recording activity measurements.

\subsection{Comprehensive understanding of enzymatic regulation by $S M$ studies}

Comprehensive description of the mechanism underlying enzymatic activity regulation is critical for understanding and controlling a spectrum of biological 
functions. ${ }^{135,149-154}$ To date our knowledge on regulation primarily relies on bulk measurements, over the last years however emerging single molecule measurements have provided insightful new knowledge of the molecular mechanism underlying regulation. Here we will outline the importance of understanding the mechanistic origin of regulation, the data and insights obtained from the few single molecule studies.

The two prevailing mechanisms underlying allosteric regulation as well as molecular recognition for multimeric proteins are the Koshland, Nemethy, and Filmer model $(\mathrm{KNF})^{155}$ and the Monod, Wyman, and Changeux (MWC) model, ${ }^{150,156}$ though in some cases mixed mechanisms have been reported. ${ }^{67,135,157,158}$ The KNF model posits that effector interactions on one multimeric enzyme binding site sequentially "induce" new conformational states which are more complementary to its binding partner on the rest of the monomers, thus affecting their activity. The MWC model on the other hand proposed multimeric enzymes to sample reversible discrete conformational states assumed to have well-defined activities and are redistributed by regulatory interactions. The modern view of allosteric regulation, and molecular recognition in general, has evolved to the "conformational selection" and "induced fit" models that also account for monomeric proteins. Bulk measurements cannot directly measure the inherent activity of each conformational state. It is difficult to identify whether regulatory cofactors (a) redistribute a preexisting conformational equilibrium without changing the inherent activity of each conformational state, (b) induce a new conformational state with a corresponding inherent activity or (c) operate via a convolution of both mechanisms (a) and (b).

To resolve this we recently followed for the first time regulation of enzymatic catalysis at the single molecule level. ${ }^{64}$ We examined the effect of regulation on functional states directly, arguing in addition that in the case of activity regulation, function is a more important observable compared to structure. As a model enzyme we employed the monomeric metabolic lipase from Thermomyces lanuginosus that has an active site covered by an oligopeptide, the lid. Lid dynamics of TLL are not taking place along the reaction coordinate as recently shown for other enzymes ${ }^{39,159}$; in the open state, the enzyme's active site is substrate accessible and multiple turnovers may occur. ${ }^{152}$ Using measurements and statistical analysis of stochastic turnovers we identified the existence of two major functional states that corresponded to the enzyme's active and inactive conformational states and furthermore quantified their inherent activity and interconversion rates. Titrating the proximity of the enzyme to its allosteric regulator, the lipid membrane, we quantified for the first time how the inherent activity, as well as the equilibrium between the two states, depends on the presence of the regulatory cofactor (Fig. 5(b)). We found that regulatory interactions primarily redistribute the probability of TLL to reside on either of these states, but does not introduce a new functional state with altered activity (Fig. 5(c)).

These single molecule findings allowed for the first time quantification of the changes in the energy landscape of an enzyme upon regulation. Apart from 


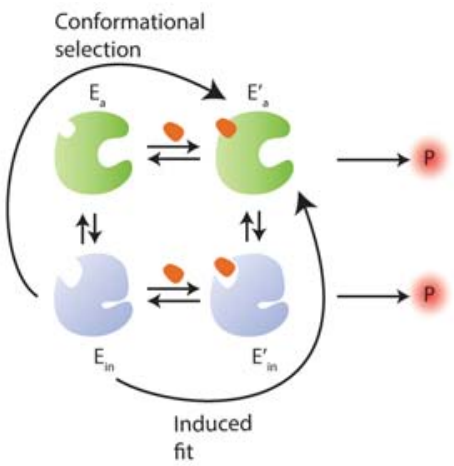

(a)
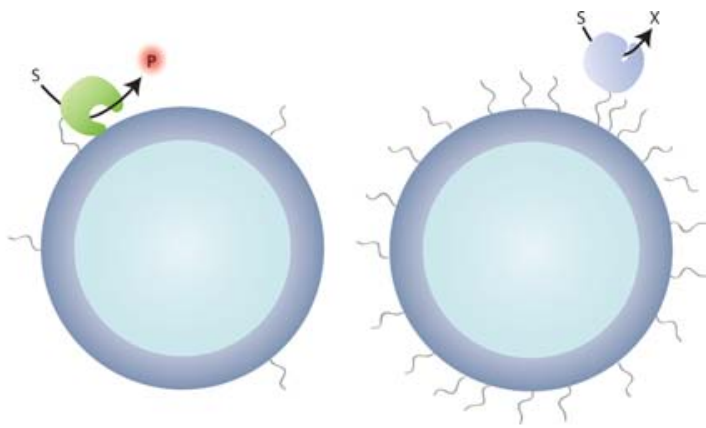

(b)

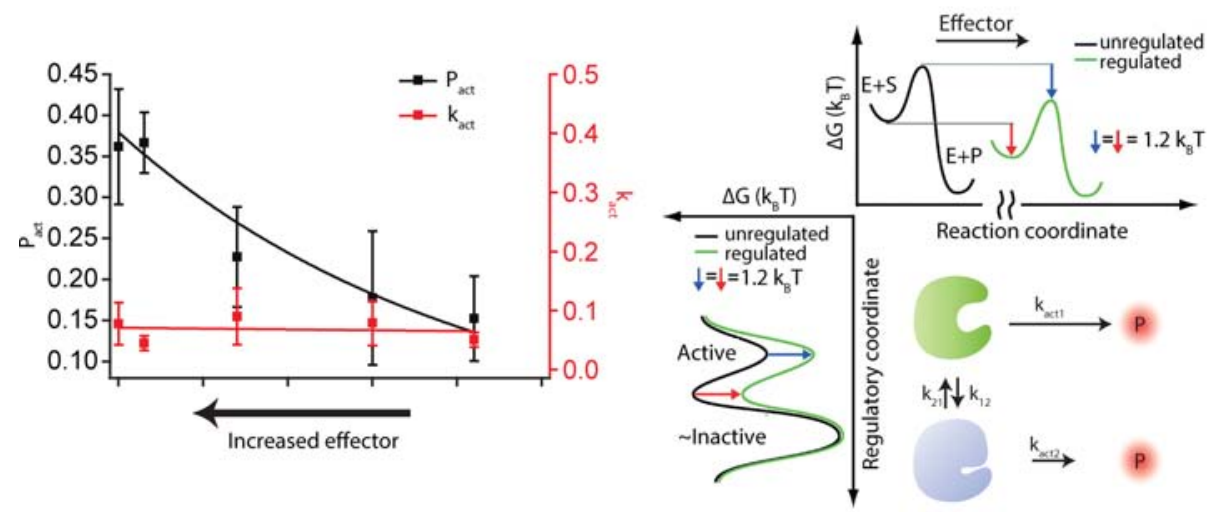

(c)

(d)

Fig. 5. (a) Thermodynamic cycle of enzymatic activity regulation by induced fit or conformational selection. Regulatory interactions for an enzyme may alter one or both of: (A) the equilibrium between the two states and (B) the inherent activity of the state. (b) Schematic representation of the methodology employed to control TLL's accessibility to its effector-bilayer. (c) Deconvolution of the parameters underlying TLL activity regulation. Controlling TLL access to the effector primarily redistributes the probability to reside in these functional states, but not to induce a new functional state. (d) Quantifications of free energy landscape remodeling for both the regulatory coordinate (vertical axis) and the reaction coordinate (horizontal axis) of TLL upon regulation as revealed by single molecule studies. Regulatory interactions equally stabilize the highly active functional state and the transition energy of the reaction. (Reprinted with permission from Ref. 64 . Copyright (2012) American Chemical Society.)

calculating energy barriers along the conformational coordinate, we quantified energy barriers along the conformational coordinate. We found regulatory interactions to equally stabilize the highly active functional state and the transition barrier along the reaction coordinate (Fig. 5(d)). These findings thus demonstrated regulation of TLL to operate via a "functional selection" mechanism.

Redistribution between a discrete number of functional states rather than changing their inherent activity upon regulation was also found in recent pioneer experiments with lysozyme tethered on carbon nanotubes field-effect transistors. ${ }^{140,146,160}$ 
During substrate processing, lysozyme undergoes an $8 \AA$, hinge-like mechanical motion creating changes in the electrostatic potential allowing continuous conformational dynamics measurements for extended periods $(\sim 10 \mathrm{~min})$. These prolonged observation times allowed the authors to identify three types of protein states: a fast fluctuating non-productive state, a slow fluctuating processing state and a closed inactive state, and furthermore to quantify their dynamics and lifetimes and how regulation by $\mathrm{pH}$ alters them. They found that regulatory interactions primarily redistribute the time spent in each state (by $\sim 3$ fold), but only moderately alter the processing rate of lysozyme. Changing the type of substrate used resulted only in slight, if any, changes in the rate of catalysis, but the time spent in rapid nonproductive motions increased radically $(\sim 6$ fold $) .{ }^{146}$

These pioneer SM measurements provided new results to the field of enzymatic activity regulation directly confirming and providing new knowledge to existing models. Firstly, they identified the existence of a discrete number of functional states. Secondly, they found regulatory interactions to remodel the energy landscape, thus changing the energy of the two states, resulting in a shift of the population distribution without altering the energy barriers that reactants have to pass along the reaction coordinate. These findings support the prevalence of a mechanism akin to "conformational selection" over the "induced fit" hypothesis. Though much remains to be learnt for an elaborate understanding of regulatory processes, we anticipate SM kinetics to become an indispensible component in this field.

\section{Conclusion and Future Directions}

Great strides have been made in characterizing enzyme dynamics function and regulation as well as in introducing the protein energy landscape, but a full mechanistic understanding of these processes remains in its infancy. Single molecule studies have emerged as an indispensable tool to provide this missing mechanistic insight on protein function. Extracting the correct model from noisy single molecule on/off trajectories is quite complicated and in some cases controversies occur. ${ }^{8,65,142}$ Recent efforts however have provided novel solutions on noise reduction and simple model development and maximum likelihood optimization, ${ }^{62,68,69,161}$ some of which are extensively discussed in this Special Issue by Flomenbom ${ }^{45}$ and by Sachs. ${ }^{46}$ It would be enticing to efficiently combine current advances in data treatment with reduced mathematical modeling and simulated data to unambiguously convert a single enzyme trajectory to a unique mechanism that can generate it. Despite the immense methodological advances in single molecule studies, the path to understanding protein function in vivo has been arduous and there is still a gap between the in vitro single molecular studies and the cellular level interactions. In vivo proteins experience a vast number of interactions with a spectrum of small molecules, protein partners and membranes, while SM studies sample only a limited number of observables at a time. Bridging this gap requires powerful new methods combining single molecule readout in native "in vivo-like" conditions and parallel 
screening of biomolecular interactions. The development of high-density microarrays of biomimetic scaffolds such as liposomes ${ }^{64,100,105,128,129}$ or nanodiscs ${ }^{162,163}$ for single molecule studies could be a novel way to implement screening of biochemical properties, molecular function or protein-effector interaction. The massive parallel redoubt $\left(10^{3}-10^{4}\right.$ liposomes per frame) of such arrays could allow direct observation of multiple protein native states and quantification of their activity abundance and redistribution by regulatory inputs.

A comprehensive description of how proteins operate in real time requires connection between different timescales and complementary interdisciplinary approaches. Single molecule kinetic measurements combined with NMR spectrometry, computational studies and mathematical modeling as well as with single molecule FRET measurements and patch clamp techniques, that are discussed in this issue by Flomenbom, ${ }^{45} \mathrm{Sach}^{46}$ and $\mathrm{Lu}^{44}$ respectively, are beginning to emerge. Coalescing the readout of these complementary approaches may enable a holistic understanding of the orchestrated enzyme dynamics and functional regulation interactions that underlie and tightly control cellular functions.

\section{Acknowledgments}

This work was supported by the UNIK research initiative of the Danish Ministry of Science, Technology and Innovation through the Center for Synthetic Biology at the University of Copenhagen, the Lundbeck Foundation Center of Excellence "Biomembranes in Nanomedicine" and the UCPH Excellence Programme for Interdisciplinary Research provided by University of Copenhagen.

\section{References}

1. H. Frauenfelder, S. G. Sligar and P. G. Wolynes, Science 254, 1598 (1991).

2. K. Henzler-Wildman and D. Kern, Nature 450, 964 (2007).

3. R. G. Smock and L. M. Gierasch, Science 324, 198 (2009).

4. H. Frauenfelder, G. Chen, J. Berendzen et al., Proc. Natl. Acad. Sci. USA 106, 5129 (2009).

5. S. A. Adcock and J. A. McCammon, Chem. Rev. 106, 1589 (2006).

6. M. Karplus and J. A. McCammon, Nat. Struct. Biol. 9, 646 (2002).

7. D. E. Shaw, P. Maragakis, K. Lindorff-Larsen et al., Science 330, 341 (2010).

8. A. V. Pisliakov, J. Cao, S. C. L. Kamerlin and A. Warshel, Proc. Natl. Acad. Sci. USA 106, 17359 (2009).

9. K. A. Henzler-Wildman, M. Lei, V. Thai et al., Nature 450, 913 (2007).

10. B. Rotman, Proc. Natl. Acad. Sci. USA 47, 1981 (1961).

11. M. J. Comstock, T. Ha and Y. R. Chemla, Nat. Methods 8, 335 (2011).

12. A. Engel and D. J. Muller, Nat. Struct. Biol. 7, 715 (2000).

13. T. E. Fisher, P. E. Marszalek and J. M. Fernandez, Nat. Struct. Biol. 7, 719 (2000).

14. Y. F. Dufrene, Nat Rev. Microbiol. 6, 674 (2008).

15. D. J. Muller and Y. F. Dufrene, Nat. Nanotech. 3, 261 (2008).

16. D. J. Muller, J. Helenius, D. Alsteens and Y. F. Dufrene, Nat. Chem. Biol. 5, 383 (2009).

17. K. C. Neuman and A. Nagy, Nat. Methods 5, 491 (2008). 
18. L. B. Oddershede, Nat. Chem. Biol. 8, 879 (2012).

19. W. J. Greenleaf, M. T. Woodside and S. M. Block, High-resolution, single-molecule measurements of biomolecular motion, in Annu. Rev. Biophys. Biomol. Struct. 36, 171-190 (2007).

20. T. Ha, A. G. Kozlov and T. M. Lohman, Annu. Rev. Biophys. 41, 295 (2012).

21. P. V. Cornish and T. Ha, ACS Chem. Biol. 2, 53 (2007).

22. M. Gudmand, S. Rocha, N. S. Hatzakis et al., Biophys. J. 98, 1873 (2010).

23. S. Rocha, J. A. Hutchison, K. Peneva et al., Chemphyschem 10, 151 (2009).

24. W. E. Moerner, Proc. Natl. Acad. Sci. USA 104, 12596 (2007).

25. F. Pinaud, S. Clarke, A. Sittner and M. Dahan, Nat. Methods 7, 275 (2010).

26. H. Yang, Curr. Opin. Chem. Biol. 14, 3 (2010).

27. C. Veigel and C. F. Schmidt, Nat. Rev. Mol. Cell. Biol. 12, 163 (2011).

28. H. Engelkamp, N. S. Hatzakis, J. Hofkens et al., Chem. Commun. (Camb.) 935 (2006).

29. V. I. Claessen, H. Engelkamp, P. C. M. Christianen et al., Single-biomolecule kinetics: The art of studying a single enzyme, in Annu. Rev. Anal. Chem., Vol. 3, eds. E. S. Yeung and R. N. Zare, 319 (2010).

30. R. D. Smiley and G. G. Hammes, Chem. Rev. 106, 3080 (2006).

31. W. Min, B. P. English, G. B. Luo et al., Acc. Chem. Res. 38, 923 (2005).

32. N. G. Walter, C. Y. Huang, A. J. Manzo and M. A. Sobhy, Nat. Methods 5, 475 (2008).

33. P. Tinnefeld and M. Sauer, Angew. Chem. Int. Ed. 44, 2642 (2005).

34. S. Kalinin, T. Peulen, S. Sindbert et al., Nat. Methods 9, 1218 (2012).

35. T. J. Ha, A. Y. Ting, J. Liang et al., Proc. Natl. Acad. Sci. USA 96, 893 (1999).

36. Y. F. Zhao, D. Terry, L. Shi et al., Nature 465, 188 (2010).

37. Y. F. Zhao, D. S. Terry, L. Shi et al., Nature 474, 109 (2011).

38. Y. Santoso, C. M. Joyce, O. Potapova et al., Proc. Natl. Acad. Sci. USA 107, 715 (2010).

39. K. A. Henzler-Wildman, V. Thai, M. Lei et al., Nature 450, 838 (2007).

40. C. Joo, H. Balci, Y. Ishitsuka, C. Buranachai and T. Ha, Annu. Rev. Biochem. 77, 51 (2008).

41. M. J. Ruedas-Rama, J. M. Alvarez-Pez and A. Orte, Biophys. Rev. Lett. 8, 161-190 (2013).

42. E. Perozo, Nat. Rev. Mol. Cell. Biol. 7, 109 (2006).

43. J. Dunlop, M. Bowlby, R. Peri, D. Vasilyev and R. Arias, Nat. Rev. Drug Discov. 7, 358 (2008).

44. H. P. Lu, Probing single-molecule ion channel dynamics by combined patch-clamp single-molecule imaging microscopy, to appear in Biophys. Rev. Lett.

45. O. Flomenbom, Biophys. Rev. Lett. 8, 109-136 (2013).

46. C. Nicholai and F. Sach, Biophys. Rev. Lett. 8, 191-211 (2013).

47. H. P. Lu, L. Y. Xun and X. S. Xie, Science 282, 1877 (1998).

48. Q. F. Xue and E. S. Yeung, Nature 373, 681 (1995).

49. D. B. Craig, E. A. Arriaga, J. C. Y. Wong, H. Lu and N. J. Dovichi, J. Am. Chem. Soc. 118, 5245 (1996).

50. J. Shi, B. A. Palfey, J. Dertouzos et al., J. Am. Chem. Soc. 126, 6914 (2004).

51. J. Shi, J. Dertouzos, A. Gafni, D. Steel and B. A. Palfey, Proc. Natl. Acad. Sci. USA 103, 5775 (2006).

52. J. R. Brender, J. Dertouzos, D. P. Ballou et al., J. Am. Chem. Soc. 127, 18171 (2005).

53. J. Shi, A. Gafni and D. Steel, Eur. Biophys. J. Biophy. 35, 633 (2006).

54. N. Dan, Curr. Opin. Colloid Interface Sci. 12, 314 (2007). 
55. S. Kuznetsova, G. Zauner, T. J. Aartsma et al., Proc. Natl. Acad. Sci. USA 105, 3250 (2008).

56. P. T. R. Rajagopalan, Z. Q. Zhang, L. McCourt et al., Proc. Natl. Acad. Sci. USA 99, 13481 (2002).

57. Z. Q. Zhang, P. T. R. Rajagopalan, T. Selzer, S. J. Benkovic and G. G. Hammes, Proc. Natl. Acad. Sci. USA 101, 2764 (2004).

58. R. B. Altman, D. S. Terry, Z. Zhou et al., Nat. Methods 9, 68 (2012).

59. R. B. Altman, Q. Zheng, Z. Zhou et al., Nat. Methods 9, 428 (2012).

60. L. Edman, Z. Foldes-Papp, S. Wennmalm and R. Rigler, Chem. Phys. 247, 11 (1999).

61. K. Velonia, O. Flomenbom, D. Loos et al., Angew. Chem. Int. Ed. 44, 560 (2005).

62. O. Flomenbom, K. Velonia, D. Loos et al., Proc. Natl. Acad. Sci. USA 102, 2368 (2005).

63. N. S. Hatzakis, H. Engelkamp and K. Velonia, Chem. Commun. (Camb.) 2012 (2006).

64. N. S. Hatzakis, L. Wei, S. K. Jorgensen et al., J. Am. Chem. Soc. 134, 9296 (2012).

65. G. De Cremer, M. B. J. Roeffaers, M. Baruah et al., J. Am. Chem. Soc. 129, 15458 (2007).

66. B. P. English, W. Min, A. M. van Oijen et al., Nat. Chem. Biol. 2, 87 (2006).

67. B. Y. Ma and R. Nussinov, Curr. Opin. Chem. Biol. 14, 652 (2010).

68. O. Flomenbom and R. J. Silbey, Proc. Natl. Acad. Sci. USA 103, 10907 (2006).

69. O. Flomenbom and R. J. Silbey, Phys. Rev. E 78, 066105 (2008).

70. P. Zhu and H. G. Craighead, Annu. Rev. Biophys. 41, 269 (2012).

71. M. J. Levene, J. Korlach, S. W. Turner et al., Science 299, 682 (2003).

72. J. Eid, A. Fehr, J. Gray et al., Science 323, 133 (2009).

73. S. Uemura, C. E. Aitken, J. Korlach et al., Nature 464, 1012 (2010).

74. K. T. Samiee, J. M. Moran-Mirabal, Y. K. Cheung and H. G. Craighead, Biophys. J. 90, 3288 (2006).

75. J. M. Moran-Mirabal, A. J. Torres, K. T. Samiee, B. A. Baird and H. G. Craighead, Nanotechnology 18, 195101 (2007).

76. D. S. Talaga, W. L. Lau, H. Roder et al., Proc. Natl. Acad. Sci. USA 97, 13021 (2000).

77. M. Friedel, A. Baumketner and J. E. Shea, Proc. Natl. Acad. Sci. USA 103, 8396 (2006).

78. R. H. Goldsmith, L. C. Tabares, D. Kostrz et al., Proc. Natl. Acad. Sci. USA 108, 17269 (2011).

79. L. M. Ying and X. S. Xie, J. Phys. Chem. B 102, 10399 (1998).

80. I. Rasnik, S. A. Mckinney and T. Ha, Acc. Chem. Res. 38, 542 (2005).

81. A. E. Cohen and A. P. Fields, Acs. Nano. 5, 5296 (2011).

82. H. C. Kolb, M. G. Finn and K. B. Sharpless, Angew. Chem. Int. Ed. 40, 2004 (2001).

83. A. J. T. Dirks, S. S. van Berkel, N. S. Hatzakis et al., Chem. Commun. (Camb.) $4172(2005)$.

84. J. L. Brennan, N. S. Hatzakis, T. R. Tshikhudo et al., Bioconjug. Chem. 17, 1373 (2006).

85. M. Meldal and C. W. Tornoe, Chem. Rev. 108, 2952 (2008).

86. A. E. Cohen and W. E. Moerner, Proc. Natl. Acad. Sci. USA 103, 4362 (2006).

87. R. H. Goldsmith and W. E. Moerner, Nat. Chem. 2, 179 (2010).

88. S. Bockenhauer, A. Furstenberg, X. J. Yao, B. K. Kobilka and W. E. Moerner, J. Phys. Chem. B 115, 13328 (2011).

89. S. Veshaguri, M. Tutkus, C. V. Lundgaard et al., Biophys. J. 104, 61A (2013).

90. M. Comellas-Aragones, H. Engelkamp, V. I. Claessen et al., Nat. Nanotech. 2, 635 (2007).

91. I. J. Minten, V. I. Claessen, K. Blank et al., Chem. Sci. 2, 358 (2011). 
92. W. H. Tan and E. S. Yeung, Anal. Chem. 69, 4242 (1997).

93. D. M. Rissin, H. H. Gorris and D. R. Walt, J. Am. Chem. Soc. 130, 5349 (2008).

94. Z. H. Li, R. B. Hayman and D. R. Walt, J. Am. Chem. Soc. 130, 12622 (2008).

95. D. R. Walt, Anal. Chem. 85, 1258 (2013).

96. H. H. Gorris, D. M. Rissin and D. R. Walt, Proc. Natl. Acad. Sci. USA 104, 17680 (2007).

97. H. H. Gorris and D. R. Walt, J. Am. Chem. Soc. 131, 6277 (2009).

98. S. M. Christensen and D. Stamou, Soft Matter 3, 828 (2007).

99. P. Walde and S. Ichikawa, Biomol. Eng. 18, 143 (2001).

100. N. S. Hatzakis, V. K. Bhatia, J. Larsen et al., Nat. Chem. Biol. 5, 835 (2009).

101. V. K. Bhatia, N. S. Hatzakis and D. Stamou, Semin. Cell Dev. Biol. 21, 381 (2010).

102. V. K. Bhatia, K. L. Madsen, P. Y. Bolinger et al., EMBO J. 28, 3303 (2009).

103. V. Früh, A. P. IJzerman and G. Siegal, Chem. Rev. 111, 640 (2011).

104. S. K. Jorgensen, D. McMillan, L. Krzeminski et al., Biophys. J. 104, 277A (2013).

105. S. M. Christensen, P. Y. Bolinger, N. S. Hatzakis, M. W. Mortensen and D. Stamou, Nat. Nanotech. 7, 51 (2012).

106. B. Stadler, R. Chandrawati, A. D. Price et al., Angew. Chem. Int. Ed. 48, 4359 (2009).

107. K. Kurihara, M. Tamura, K. Shohda, T. Toyota, K. Suzuki and T. Sugawara, Nat. Chem. 3, 775 (2011).

108. M. Michel, M. Winterhalter, L. Darbois et al., Langmuir 20, 6127 (2004).

109. D. M. Vriezema, M. C. Aragones, J. Elemans et al., Chem. Rev. 105, 1445 (2005).

110. D. Stamou, C. Duschl, E. Delamarche and H. Vogel, Angew. Chem. Int. Ed. 42, 5580 (2003).

111. K. Hosoda, T. Sunami, Y. Kazuta et al., Langmuir 24, 13540 (2008).

112. M. Karlsson, M. Davidson, R. Karlsson et al., Annu. Rev. Phys. Chem. 55, 613 (2004).

113. E. Boukobza, A. Sonnenfeld and G. Haran, J. Phys. Chem. B 105, 12165 (2001).

114. E. Rhoades, E. Gussakovsky and G. Haran, Proc. Natl. Acad. Sci. USA 100, 3197 (2003).

115. B. Okumus, T. J. Wilson, D. M. J. Lilley and T. Ha, Biophys. J. 87, 2798 (2004).

116. J. Y. Lee, B. Okumus, D. S. Kim and T. J. Ha, Proc. Natl. Acad. Sci. USA 102, 18938 (2005).

117. M. Pirchi, G. Ziv, I. Riven et al., Nat. Commun. 2, 1 (2011).

118. I. Cisse, B. Okumus, C. Joo and T. J. Ha, Proc. Natl. Acad. Sci. USA 104, 12646 (2007).

119. J. W. Szostak, D. P. Bartel and P. L. Luisi, Nature 409, 387 (2001).

120. P. Y. Bolinger, D. Stamou and H. Vogel, J. Am. Chem. Soc. 126, 8594 (2004).

121. P. Y. Bolinger, D. Stamou and H. Vogel, Angew. Chem. Int. Ed. 47, 5544 (2008).

122. D. M. Vriezema, P. M. L. Garcia, N. S. Oltra et al., Angew. Chem. Int. Ed. 46, 7378 (2007).

123. M. T. Reetz, Angew. Chem. Int. Ed. 50, 138 (2011).

124. R. D. Schmid and R. Verger, Angew. Chem. Int. Ed. 37, 1609 (1998).

125. N. S. Hatzakis, D. Daphnomili and I. Smonou, J. Mol. Catal. B: Enzym. 21, 309 (2003).

126. N. S. Hatzakis and I. Smonou, Bioorg. Chem. 33, 325 (2005).

127. Y. G. Shi and P. Burn, Nat. Rev. Drug Discov. 3, 695 (2004).

128. E. Elizondo, J. Larsen, N. S. Hatzakis et al., J. Am. Chem. Soc. 134, 1918 (2012).

129. J. Larsen, N. S. Hatzakis and D. Stamou, J. Am. Chem. Soc. 133, 10685 (2011). 
130. P. M. Bendix, M. S. Pedersen and D. Stamou, Proc. Natl. Acad. Sci. USA 106, 12341 (2009).

131. H. Yang, G. B. Luo, P. Karnchanaphanurach et al., Science 302, 262 (2003).

132. C. B. Li, H. Yang and T. Kornatsuzaki, Proc. Natl. Acad. Sci. USA 105, 536 (2008).

133. G. M. Lee and C. S. Craik, Science 324, 213 (2009).

134. D. D. Boehr, H. J. Dyson and P. E. Wright, Chem. Rev. 106, 3055 (2006).

135. D. D. Boehr, R. Nussinov and P. E. Wright, Nat. Chem. Biol. 5, 789 (2009).

136. D. D. Boehr, D. McElheny, H. J. Dyson and P. E. Wright, Proc. Natl. Acad. Sci. USA 107, 1373 (2010).

137. H. P. Lerch, R. Rigler and A. S. Mikhailov, Proc. Natl. Acad. Sci. USA 102, 10807 (2005).

138. X. S. Xie and H. P. Lu, J. Biol. Chem. 274, 15967 (1999).

139. Q. Chen, R. Groote, H. Schonherr and G. J. Vancso, Chem. Soc. Rev. 38, 2671 (2009).

140. Y. K. Choi, I. S. Moody, P. C. Sims et al., Science 335, 319 (2012).

141. H. P. Lerch, A. S. Mikhailov and B. Hess, Proc. Natl. Acad. Sci. USA 99, 15410 (2002).

142. T. G. Terentyeva, H. Engelkamp, A. E. Rowan et al., ACS Nano 6, 346 (2012).

143. T. G. Terentyeva, J. Hofkens, T. Komatsuzaki, K. Blank and C. B. Li, J. Phys. Chem. B 117, 1252 (2013).

144. O. Flomenbom, Making it possible: Constructing a reliable mechanism from a finite trajectory, in Single-Molecule Biophysics: Experiment and Theory, Vol. 146, eds. T. Komatsuzaki, M. Kawakami, S. Takahashi, H. Yang and R. J. Silbey, 367 (2012).

145. M. B. J. Roeffaers, G. De Cremer, H. Uji-i et al., Proc. Natl. Acad. Sci. USA 104, 12603 (2007).

146. Y. Choi, I. S. Moody, P. C. Sims et al., J. Am. Chem. Soc. 134, 2032 (2012).

147. R. Polakowski, D. B. Craig, A. Skelley and N. J. Dovichi, J. Am. Chem. Soc. 122, 4853 (2000).

148. S. V. Solomatin, M. Greenfeld, S. Chu and D. Herschlag, Nature 463, 681 (2010).

149. L. Y. Wang, A. Pulk, M. R. Wasserman et al., Nat. Struct. Mol. Biol. 19, 957 (2012).

150. J. P. Changeux and S. J. Edelstein, Science 308, 1424 (2005).

151. M. P. Bokoch, Y. Z. Zou, S. G. F. Rasmussen et al., Nature 463, 108 (2010).

152. J. Kuriyan and D. Eisenberg, Nature 450, 983 (2007).

153. S. R. Tzeng and C. G. Kalodimos, Nature 462, 368 (2009).

154. N. M. Goodey and S. J. Benkovic, Nat. Chem. Biol. 4, 474 (2008).

155. D. E. Koshland, Proc. Natl. Acad. Sci. USA 44, 98 (1958).

156. J. Monod, J. Wyman and J. P. Changeux, J. Mol. Biol. 12, 88 (1965).

157. G. G. Hammes, Y. C. Chang and T. G. Oas, Proc. Natl. Acad. Sci. USA 106, 13737 (2009).

158. S. M. Sullivan and T. Holyoak, Proc. Natl. Acad. Sci. USA 105, 13829 (2008).

159. D. D. Boehr, D. McElheny, H. J. Dyson and P. E. Wright, Science 313, 1638 (2006).

160. T. J. Olsen, Y. Choi, P. C. Sims et al., J. Am. Chem. Soc. 135, 7855 (2013).

161. O. Flomenbom, J. Hofkens, K. Velonia et al., Chem. Phys. Lett. 432, 371 (2006).

162. M. Wadsater, T. Laursen, A. Singha et al., J. Biol. Chem. 287, 34596 (2012).

163. A. Nath, A. J. Trexler, P. Koo et al., Single-Molecule Fluorescence Spectroscopy Using Phospholipid Bilayer Nanodiscs, in Methods in Enzymology, Vol. 472: Single Molecule Tools, Pt. A: Fluorescence Based Approaches, ed. N. G. Walter, 89 (2010). 